\title{
Extraperitoneal Antegrade vs Transperitoneal Open Radical Cystectomy: Single Center Experiences with 200 Cases
}

\author{
Orkunt Özkaptan, Alkan Çubuk*, Erdinç Dinçer, Ahmet Şahan, Alper Kafkaslí and Oktay Akça \\ Department of Urology, Kartal Lutfi Kirdar Training and Research Hospital, Kartal, Istanbul, Turkey
}

Received 4 February 2020

Accepted 1 April 2020

Pre-press 23 April 2020

Published 11 June 2020

\begin{abstract}
.
BACKGROUND: Radical cystectomy (RC) is one of the most complex surgeries and has a high rate of morbidity. Gastrointestinal complications are the most common type of complications. To reduce these complications some modifications have been described.

OBJECTIVE: To evaluate perioperative outcomes of our extraperitoneal antegrade RC technique (EARTC), where the peritoneum is opened at the end of cystectomy just before of ileal reconstruction.

METHODS: Group 1 included 120 patients who were operated with a standard RC technique and Group 2 included 80 patients who were operated with the EARC technique in this study. Groups were compared according to preoperative variables including patient characteristics, perioperative parameters, pathologic data, and postoperative overall and gastrointestinal complications.

RESULTS: There were no significant differences between the two groups in terms of preoperative characteristics and mean operative time. The group 1 has longer time for the exposure of abdominal cavity to the atmosphere $(p<0.01)$. Hospitalization time was significantly lower in Group $2(p<0.01)$. Concerning the rate of 90 -day overall perioperative complication, no statistically significant difference was determined between the groups. Gastrointestinal complication was significantly higher in Group 1 ( $p: 0.048)$. The average number of removed lymph nodes was similar between the groups $(p: 0.85)$. The time for recovery of bowel function, the time for passage of stool and the rate of postoperative ileus were significantly lower in Group $2(p<0.01, p<0.01$ and $p<0.043)$ respectively).

CONCLUSIONS: EARC provides advantages over the standard technique in terms of gastrointestinal symptoms and poses no disadvantage when the oncological outcome and operative difficulty were considered.
\end{abstract}

Keywords: Bladder cancer, radical cystectomy, ileus, complication, extra-peritoneal

\section{INTRODUCTION}

Radical cystectomy (RC) is the standard treatment for localized or locally advanced invasive bladder cancer and high-risk superficial disease [1]. It pro-

\footnotetext{
*Correspondence to: Alkan Çubuk, Department of Urology, Kartal Training and Research Hospital, Cevizli Mh., Şemsi Denizer Cad. E-5 Karayolu Cevizli Mevkii, 34890, Kartal, İstanbul, Turkey. Tel.: +90 506 2984762; E-mail: alkancubuk@ hotmail.com.
}

vides excellent local cancer control with the lowest pelvic recurrence rates and $50 \%$ to $70 \%$ 5-years cancer-specific survival rates [2].On the other hand, this procedure is one of the most complex and challenging surgeries in urology. The complexity of RC and diversion results in a high risk of perioperative morbidity. The perioperative complication rate in RC in studies using standard reporting systems of $\mathrm{RC}$ varies between $40 \%$ and $64 \%$ in the literature. 
[3-5].The most common complication categories are gastrointestinal (29\%), infectious (25\%) and woundrelated $(15 \%)$, respectively [3].

Ileus is the most common type of gastrointestinal complications (GC) [3]. The rate of ileus varies around $17.8-23 \%[3,6]$. The reasons for the relative high GC rates may be prolonged exposure of the bowels to the atmosphere, tactile manipulation of the bowels, and the use of an ileal segment for urinary diversion.

The standard RC is performed with a transperitoneal technique where the peritoneum is opened in the early period of the procedure, and the bladder is dissected in an antegrade fashion [7]. In 1999, Kulkarni et al. introduced an ascending extraperitoneal technique with extraperitonealization of the ileal bladder in order to reduce morbidity by separating the extra- and intraperitoneal healing processes [8]. However, Roth et al. mentioned the concern about the radicalness of surgery in extraperitoneal cystectomy of this technique [9].

In the last years, we have performed an extraperitoneal antegrade RC technique (EARC) where the peritoneum is opened at the end of cystectomy just before of ileal reconstruction. We aimed to reduce the tactile manipulation of the bowels and the contact of the bowels with the atmosphere. In the current study, we compared the perioperative outcomes and complications of the conventional RC technique with our modified RC technique.

\section{MATERIAL AND METHOD}

The data from 200 patients who underwent $\mathrm{RC}$ and ileal conduit for bladder cancer with two different open approaches between April 2013 and December 2019 were included in the study. Data of the patients were recorded prospectively in our electronic database and reviewed retrospectively for the study. In order to provide a homogeneous study population, patients who underwent an orthototopic neobladder were not included in this study. The study was approved by Institutional Review Board (IRB Number: 2019-03331). Due to the retrospective nature of the study, only written consent was obtained from the patients.

All procedures were performed by a single senior surgeon who is highly experienced in urooncologic surgery. The first 120 patients were operated on with a standard RC technique between 2013 and 2016 (Group 1). The final eighty patients were operated on with the EARC technique in 2016-2019 (Group 2). A specific matching procedure was not applied.

Preoperative variables including patient characteristics, perioperative parameters, pathologic data, and postoperative overall and gastrointestinal complications were evaluated. Perioperative complications were classified according to the Martin criteria [10].

\section{Preoperative preparation}

All patients received a rectal enema one day before the surgery. Solid food fasting was applied for two days before the operation. Third-generation cephalosporin was administered for prophylaxis against infection until the removal of all catheters and metronidazole for three days. Low weight heparin and elastic compressive stockings were used against thromboembolic events in all patients. An epidural catheter was placed preoperatively, and combined anesthesia (general and epidural) was given intraoperatively.

The perioperative outcomes included the duration of surgery (defined as skin incision to skin closure time in both procedures), estimated blood loss (EBL), hospitalization time (HT) and transfusion rates (TR). The duration of bowel exposure to the atmosphere was also recorded.

Patients were monitored in the interdisciplinary ICU on the first postoperative day, routinely. Afterwards, the patients were followed-up and treated according to our standardized cystectomy pathways. Postoperatively, all patients had a nasogastric tube left in place until the first passage of flatus. Daily clinical examinations and laboratory monitoring were performed in a standard fashion. Follow-up during the third-month visits consisted of a physical examination, blood tests, urine culture and abdominopelvic computed tomography. All complications within 90 days were recorded and graded according to the five-grade modified version of the original ClavienDindo grading system [11]. Major complications were defined as grade 3 to 5 while minor or no complications as grade 0,1 , and 2 .

Ileus was defined as abdominal distension together with nausea and vomiting, requiring cessation of oral intake and intravenous fluid support and/or nasogastric tube placement; or the intolerance of oral intake by postoperative day 4, resulting in patient fasting with/without nasogastric tube placement or antiemetic medication administration [12]. The time of recovery of normal bowel 
activity and mean stool passage time was also evaluated.

\section{Surgical technique}

\section{The standard RC technique}

In the standard RC technique the peritoneum was opened at the beginning of the surgery as described previously $[1,2]$. An ileal conduit using the Wallace technique for uretero-intestinal anastomosis was performed as diversion. A routine lymph node dissection including aortic bifurcation, presacral, common iliac, external iliac, internal iliac and obturator nodes was performed for both groups.

\section{EARC technique}

The patient is placed in a 15-degree Trendelenburg position. After an infra-umbilical incision the Retzius space is entered. The pelvic peritoneum is gently pushed cephalad at the level of the vas deferens on each side. The vas deferens were dissected and cut on both sides. The avascular plane (paravesical space) on each side of the prostate and bladder is created. Then bilateral extended pelvic lymphadenectomy is performed. After cleaning off the periprostatic fatigue tissue, the endopelvic fascia is incised on either side. After the levator muscles are separated from the prostate, the apex of the prostate is identified. The deep dorsal vein (DDV) is transacted and cut. The anterior urethral wall is incised with cautery. A right-angled clamp is closely passed along, and the posterior urethral wall is cut. The neurovascular bundles are mobilized from the level of the bladder neck to the urethra. The posterior side of the prostate is dissected and mobilized cephalad by traction on the Foley catheter. Afterwards, the lateral pedicles of the prostate are divided. The Denovilliers fascia containing the seminal vesicles is released from the rectum. Superior vesical vessels are identified and cut. Afterwards, the ureters are identified and are separated. After the urachus is dissected at the level of the umbilicus, the specimen containing the peritoneal layer on the posterior bladder is dissected off. The peritoneum is usually not opened until this part of the procedure.

\section{Statistical analysis}

Baseline characteristics and overall outcomes were summarized as the mean and standard deviation (SD) for normally distributed continuous variables, and frequencies and percentages for categorical vari- ables. To assess the differences between the two groups according to the time of stool passage, recovery of bowel activity, duration of bowel exposure to the atmosphere and perioperative outcome, the independent sample $T$-test was used. Differences between the two groups for overall and gastrointestinal complication rates were assessed using the Chi-square test or Fisher's exact test. SPSS version 20.0 (Chicago, Il, USA) was used for statistical analyses. A $P$ value of $<0.05$ was considered statistically significant.

\section{RESULTS}

The average age at diagnosis was 65 (38-85) for Group 1 and 65 (41-86) for Group 2, respectively ( $p: 0.68)$. There were no significant differences among the two groups in terms of age, BMI, previous history of abdominal surgery, clinical stage of bladder cancer and Charlson comorbidity index. Preoperative clinical and pathological features of the patients were similar between the two groups (Table 1).

There was no difference between the two techniques in terms of the operative time. The mean operative time was $230(210-450)$ for Group 1 and 240 (215-510) for Group 2, respectively. The duration for the exposure of the abdominal cavity to the atmosphere during the operation was statistically significantly longer in Group 1 (228 (209-448) minutes vs 68 (215-510) minutes; $p<0.01)$. Hospitalization time (HT) was statistically significantly lower in Group $2(p<0.01)$. The median volume of EBL was not different among the groups; it was 650 (320-3100) $\mathrm{ml}$ for Group 1 and $700(350-2900) \mathrm{ml}$ for Group 2, respectively ( $p: 0.82$ ) (Table 2).

Concerning the rate of 90-day overall perioperative complication, no statistically significant difference was determined between the two groups (Table 3). A total of 191 complications developed in 128 $(64 \%)$ of the 200 patients. Sixty-seven of complications were determined in the standard RC group, whereas 51 complications occurred in the modified RC group. Furthermore, we did not observe a statistically significant difference in major or minor complication rates according to Clavien classification between the groups ( $p=0.86$ and $p=0.86$; respectively). The rates of minor and major complications were $48.75 \%(\mathrm{~N}=39)$ and $15 \%(\mathrm{~N}=12)$ for Group 1 and $50 \%(\mathrm{~N}=60)$ and $14.17 \%(\mathrm{~N}=17)$ for Group 2 , respectively. A total of two patients died due to cardiovascular events. 
Table 1

Patient Characteristics

\begin{tabular}{|c|c|c|c|}
\hline Variable & Group 1 & Group 2 & $P$ value \\
\hline Patient number & 120 & 80 & \\
\hline Age, median (range) & $65(38-85)$ & $65(41-86)$ & 0.677 \\
\hline \multicolumn{4}{|l|}{ Gender, $\mathrm{N}$} \\
\hline Male & 115 & 75 & 0.639 \\
\hline Female & 10 & 5 & \\
\hline${ }^{\dagger} \mathrm{BMI}$, median (range) & $26.08(18.9-39.2)$ & $25.31(18-38.2)$ & 0.511 \\
\hline Charlson's score, median, (range) & $3(2-8)$ & $4(2-8)$ & 0.637 \\
\hline Operating time, minutes, median (range) & $230(210-450)$ & $240(215-510)$ & \\
\hline Hospitalization time, day, median (range) & $10(4-41)$ & $9(4-32)$ & $0.042 *$ \\
\hline Estimated blood loss, ml, median (range) & $650(320-3100)$ & $700(350-2900)$ & 0.41 \\
\hline \multicolumn{4}{|l|}{$\begin{array}{l}\text { Pathological stage, } \\
n(\%)\end{array}$} \\
\hline $\mathrm{pTa} / \mathrm{pTcis}$ & 7 & 7 & 0.458 \\
\hline pT1 & 7 & 7 & \\
\hline pT2 & 34 & 17 & \\
\hline pT3 & 49 & 28 & \\
\hline pT4 & 23 & 21 & \\
\hline Pathological lymph node, $n(\%)$ status & & & 0.787 \\
\hline NO & 92 & 60 & \\
\hline $\mathrm{N}+$ & 28 & 20 & \\
\hline \multicolumn{4}{|l|}{ Organ confined disease } \\
\hline${ }^{8}$ Clavien-Dindo classification, $n(\%)$ & & & 0.84 \\
\hline Major $3 \leq$ & $17(14.17)$ & $12(15)$ & \\
\hline Minor 1-2 & $60(50)$ & $39(48.75)$ & \\
\hline
\end{tabular}

Most common complications that occurred in Group 1 were gastrointestinal $(28.3 \%, \mathrm{~N}=34)$, infectious $(21.6 \%, \mathrm{~N}=21)$ and wound-related $(15.8 \%$, $\mathrm{N}=19$ ) complications. Of GC, ileus was the most common complication observed in Group 1 with a rate of $19 \%(\mathrm{~N}=23)$. In Group 2, infectious $(20 \%$, $\mathrm{N}=16)$ and wound-related $(18.75 \%, \mathrm{~N}=15)$ complications were observed the most commonly. The rate of GC was significantly higher in Group 1 (28.3\% vs $16.5 \%, p=0.048)$.

Furthermore, we compared the two groups according to ileus rates, time for recovery of bowel function and mean time for passage of stool. The time for recovery of bowel function was significantly lower in Group $2(p<0.01)$. Similarly, the difference of the passage of stool time between the groups was statistically significant $(p<0.01)$ Furthermore, the incidence of ileus between the groups differed significantly. Ileus was determined in $18.4 \%(\mathrm{~N}=23)$ of the patients in Group 1 and $5.83 \%(\mathrm{~N}=7)$ of the patients in Group 2 ( $p: 0.043$ ) (Table3).

The average number of removed lymph nodes was 19 (13-29) and $18.3(13-28)$ in Group 1 and Group 2, respectively $(p=0.85)$. Lymphoceles were not observed in any of the groups.

\section{DISCUSSION}

Radical cystectomy using a transperitoneal approach is the most common technique performed for the treatment of muscle-invasive or high-risk noninvasive bladder cancer. Even though this technique has been used as a well-established procedure over the years, the occurrence of complications is still not at a low rate. The overall complication rate of open RC, according to the results of standardized methodologies for reporting complications, varies between $49 \%$ and $64 \%$ [3, 12]. The most common complications after radical cystectomy are gastrointestinal complications, occurring in around $35 \%$ of patients [13-15]. The opening of the peritoneum and surgical manipulation of abdominal contents are known to cause the peristaltic impairment of the bowels [15]. Additionally, the surgery of the abdominal cavity which leads to the activation of a spinal reflex arc and generalized sympathetic hyperactivity results in depression of gastrointestinal motility [17]. Motility disorders, especially ileus, are frequently observed problems after the procedure. Preserving the peritoneum during the operation as long as possible might be efficacious to reduce these kinds of 
Table 2

Reporting of Complications in first 90 days After the Operation

\begin{tabular}{|c|c|c|c|}
\hline & Group 1, N (\%) & Group 2, N (\%) & $P$ value \\
\hline \multicolumn{4}{|c|}{ Early complications (first 90 postop. days) } \\
\hline Gastrointestinal Complications & $34(28.3)$ & $13(16.5)$ & 0.048 \\
\hline Ileus & $23(19.2)$ & $7(5.8)$ & $0.043^{*}$ \\
\hline Constipation & $5(4.2)$ & $3(3.7)$ & \\
\hline Diarrhea & $5(4.2)$ & $3(3.7)$ & \\
\hline Intestinal bleeding & $1(0.8)$ & 0 & \\
\hline Infection & $20(16.7)$ & $16(20)$ & 0.54 \\
\hline Urinary tract infection & $12(10)$ & $8(10)$ & \\
\hline Fever unknown origin & $4(3.3)$ & $4(5)$ & \\
\hline Urosepsis & $2(1.6)$ & $3(3.75)$ & \\
\hline Pyelonephritis & $1(0.8)$ & $1(1.25)$ & \\
\hline Gastroenteritis & $1(0.8)$ & 0 & \\
\hline Wound & $19(15.8)$ & $15(18.75)$ & 0.59 \\
\hline Wound infection/seroma & $16(13.3)$ & $13(16.25)$ & \\
\hline Wound dehiscence/evisceration & $3(2.5)$ & $2(2.5)$ & \\
\hline Genitourinary & $2(1.6)$ & $1(1.25)$ & \\
\hline Renal failure & $2(1.6)$ & $1(1.25)$ & \\
\hline Cardiac & $5(4.16)$ & $5(6.25)$ & \\
\hline Arrhythmia & $1(0.8)$ & $2(2.5)$ & \\
\hline Myocardial infarction & $1(0.8)$ & $1(1.25)$ & \\
\hline Syncope/hypotension & $3(2.5)$ & $2(2.5)$ & \\
\hline Pulmonary & $7(5.8)$ & $5(6.25)$ & \\
\hline Pneumonia & $2(1.6)$ & $1(1.25)$ & \\
\hline Respiratory distress & $1(0.8)$ & $1(1.25)$ & \\
\hline Pleural effusion & $1(0.8)$ & $1(1.25)$ & \\
\hline Atelectasis & $3(2.5)$ & $3(3.75)$ & \\
\hline Bleeding needing transfusion & $20(16.6)$ & $16(20)$ & 0.54 \\
\hline Thromboembolic events & $6(5)$ & $4(5)$ & \\
\hline Deep venous trombhosis & $3(2.5)$ & $2(2.5)$ & \\
\hline Pulmonary embolism & $3(2.5)$ & $2(2.5)$ & \\
\hline Neurological & 0 & $1(1.25)$ & \\
\hline Delirium/agitation & 0 & $1(1.25)$ & \\
\hline
\end{tabular}

Table 3

Comparison of Gastrointestinal Symptoms and Ileus Rates

\begin{tabular}{lccc}
\hline & Group 1 & Group 2 & $P$ value \\
\hline Recovery of Bowel Function, days, median (range) & $4(2-9)$ & $3(2-6)$ & $<0.01^{*}$ \\
Passage of Stool, day, median (range) & $5(2-10)$ & $4(2-6)$ & $<0.01^{*}$ \\
Ileus Rates, N (\%) & $23(19.2)$ & $7(5.8)$ & $0.043^{*}$ \\
\hline
\end{tabular}

complications. Kulkarni et al. described an extraperitoneal approach in radical cystectomy for the first time in 1999, according to which the peritoneum is only briefly opened for a short distance to construct a urinary conduit, with the aim of decreasing morbidity [8]. Another study emphasized the reperitonealization at the end of the operation to provide compartmentalization of the gastrointestinal system. [9]. In our technique, the peritoneum was entered during the construction of the ileal conduit without re-peritonealization at the end of the procedure. As a result, the duration of bowel exposure to the atmosphere is significantly shorter in our technique. Further tactile manipulation of the bowels during the surgery has been decreased.
Previous studies performing cystectomy with an extraperitoneal approach found lower ileus rates compared to the standard RC approach [18-20]. Similar to these studies, the ileus rate in our modified technique was significantly lower than that in the standard $\mathrm{RC}$ technique we performed. Another critical point was that the time for the return of bowel motility and the time of oral intake was significantly shorter in our modified technique. Consequently, hospitalization time was shorter in this group of patients. Ileus has been reported to be the most common cause of delayed recovery and as a consequence, prolonged hospitalization [13, 14, 21].

In contrast to the previous studies, where only a small part of the peritoneum is opened for ileal 
reconstruction, we excised the peritoneal layer on the posterior bladder wall en-bloc with the whole specimen. We think that this step is essential in terms of following oncological principles. Even though the risk of invasion of intraperitoneal viscera and retroperitoneal nodal disease is low, it is quite possible that a positive margin might be left on the posterior wall in the case of the presence of posterior bladder wall invasion. In contrast, Jentzmik et al. stated in their study that preserving the peritoneal layer on the posterior bladder wall is safe in terms of oncological outcomes and that peritoneal tumor infiltration could be detected on radiological evaluation [18]. Nevertheless, we think that radiological evaluation does not always provide reliable information about the occurrence of local invasion.

We are able to operate on patients with locally advanced disease, seminal vesicle invasion and bulky tumors with this modified technique while Kulkarni et al. excluded such patients from their study, where they preserved the posterior peritoneal layer [22]. The reason for this is most probably the risk of leaving positive tumor margins in such patients. In our opinion, preserving the posterior layer bears the risk of tumor residue; therefore a dorsolateral reperitonealization of the peritoneal layer at the end of the procedure for compartmentalization of the GI as described by Roth et al. is more eligible. Roth et al. achieved significantly less postoperative pain, early recovery of bowel function and fewer complications compared to patients without re-peritonealization in their study. The authors performed a standard RC with small modifications during the incision of the lateral peritoneal layer. They incised the lateral parietal peritoneum more dorsally to the external iliac vessels and peeled these vessels off ventrally so as to create bilateral peritoneal flaps. These flaps were used for the compartmentalization of the peritoneum at the end of the procedure [9].

Wound-related complications are one of the most frequent complications in RC [13]. Kulkarni concluded in their study that an infra-umbilical incision combined with the extraperitoneal RC technique led to lower wound-related complications [22]. In contrast to their study, our findings revealed no statistically significant difference according to wound-related complications. Further, an infraumbilical incision was sufficient for both techniques in all cases. There was no case in both groups where the incision had to be extended. Concordant with our results, Jentzmik et al. determined no difference in wound-related complications between extra and transperitoneal cystectomy techniques [18]. They hypothesized that contamination from the construction of the ileal neobladder or infectious urine from the not-yet fully-healed pouch might be responsible for wound-related complications.

The overall complication rate $(64 \%)$ of our study was in line with previous studies which used standardized reporting systems [3, 4, 12]. Similarly, overall complication rates were observed in both groups $(63.75 \%$ vs $63.33 \%)$ in our study.

According to variables reflecting operative difficulty, EBL and transfusion rates were not significantly different between the two groups. Furthermore, no intergroup difference was observed in operative time. Our results were compatible with previously reported larger series [3, 4]. Even though we did not use an objective scale for measuring operative difficulty for each case, we think that these two techniques do not differ in terms of operative difficulty. The operative outcomes of the two groups such as EBL, duration of surgery and TR, support this opinion.

Regarding the number of removed lymph nodes, we could not determine differences between the two groups $(p=0.85)$. Our findings coincide with a previous study that determined no difference in the extension of lymph node dissection in the extraperitoneal RC technique [18]. We think that there is no difference in the extend of lymph node dissection between the two techniques In our opinion lymph node dissection up to the aortic bifurcation in the EARC technique is not much more difficult than in the extraperitoneal approach. However, a superextended lymph dissection with an extraperitoneal might not be feasible. On the other hand, the risk for lymphoceles may increase in the extraperitoneal RC approach. Previous studies applying an extraperitoneal RC approach leaving a small fenestrae between the two compartments reported a lymphocele rate of $5.6 \%$ to $6.3 \%$, which is relatively high compared to conventional RC lymphocele rates $(0.54-3.57 \%)$ reported in the literature [3,5 and 23]. We did not observe lymphocele in both groups. We attribute the low rates of lymphoceles in both groups to the fact that we do not perform a regular ultrasound to define lymphoceles until they become symptomatic. As we incorporate the extra-and intraperitoneal space in the modified technique, the risk for the occurrence of lymphocele becomes most probably similar between the two approaches.

Some limitations should be noted in our study. First, the retrospective nature of the study is a 
limitation. Another limitation is the relatively low patient number. These facts preclude us from making any definitive conclusion from this study. Another weakness of the study is the difference in the period when each study group underwent the surgeries. More reliable results would be obtained in prospective randomized study design. Furthermore, the re-adaptation of the dorsolateral peritoneal layer might provide an additional contribution to a more favorable postoperative outcome. The fact that we did not perform the re-adaptation of the layer should be stated as another weakness of the study.

The postoperative follow-up protocol and the length of hospital stay are the other weaknesses of the study. The postoperative pathway of our study is not commonly used anymore by most high volume cystectomy centers. A current Enhanced Recovery After Surgery (ERAS) protocol should be used as postoperative pathway. Another reason for longer HS is that health care providers out of the hospitals like it is in western countries are not available in our country. Furthermore, most of the patients are referred from rural areas of our country, where complications may not be managed at state hospitals.

\section{CONCLUSION}

The ascending technique that we described in the current study may be associated with lower gastrointestinal complications, lower HT and coherently overall complication rates, whereas operative outcome may be similar to the standard RC technique. We believe this technique may provide some advantages over the standard technique in terms of gastrointestinal symptoms without causing to disadvantage in the oncological outcome and operative difficulty.

\section{ACKNOWLEDGMENTS}

The authors have no acknowledgments.

\section{FUNDING}

The authors report no funding.

\section{AUTHOR CONTRIBUTIONS}

Concept: OO, AC, ED; Design: OA, AK, AS; Control: OO, AC, OA; Data collection: ED, AK, AS; Analysis: AS, OO; Literature review: AC, AS;
Writing: OO, AC, OA; Critical review: OA, AK; References and Funding: ED, AC; Material: OA, ED.

\section{CONFLICT OF INTEREST}

The authors have no conflict of interest to report.

\section{REFERENCES}

[1] Konety BR, Allareddy V, Herr H. Complications after radical cystectomy: analysis of population-based data. Urology 2006;68:58-64.

[2] Shariat SF, Karakiewicz PI, Palapattu GS, Amiel GE, Lotan Y, Rogers CG, et al. Nomograms provide improved accuracy for predicting survival after radical cystectomy. Clin Cancer Res 2006;12:6663-76.

[3] Shabsigh A, Korets R, Vora KC, Brooks CM, Cronin AM, Savage C, et al. Defining early morbidity of radical cystectomy for patients with bladder cancer using a standardized reporting methodology. Eur Urol 2009;55:164:944-8.

[4] Fairey A, Chetner M, Metcalfe J, Moore R, Todd G, Rourke $\mathrm{K}$, et al. Associations among age, comorbidity and clinical outcomes after radical cystectomy: results from the Alberta Urology Institute radical cystectomy database. J Urol 2008;180:128-34.

[5] Lowrance WT, Rumohr JA, Chang SS, Clark PE, Smith JA Jr, Cookson MS , et al. Contemporary Open Radical Cystectomy: Analysis of Perioperative Outcomes. J Urol. 2008;179(4):1313-8.

[6] Chang SS, Cookson MS, Baumgartner RG, et al. Analysis of early complications after radical cystectomy: results of a collaborative care pathway. J Urol 2002;167:2012-6.

[7] Hautmann RE. Urinary diversion: Ileal conduit to neobladder. J Urol 2003;169:834-42.

[8] Kulkarni JN, Gulla RI, Tongaonkar HB, Kashyapi BD, Rajyaguru KB. Radical cystoprostatectomy: an extraperitoneal retrograde approach. J Urol. 1999;161(2):545-8.

[9] Roth B, Birkhäuser FD, Zehnder P, Burkhard FC, Thalmann GN, Studer UE. Readaptation of the peritoneum following extended pelvic lymphadenectomy and cystectomy has a significant beneficial impact on early postoperative recovery and complications: results of a prospective randomized trial. Eur Urol. 2011;59:204-10.

[10] Martin RC, Brennan MF, Jaques DP. Quality of complication reporting in the surgical literature. Ann Surg 2002;6:803-13.

[11] Dindo D, Demartines N, Clavien, PA. Classification of surgical complications: a new proposal with evaluation in a cohort of 6336 patients and result sofa survey. AnnSurg 2004;240:205-13.

[12] Novara G, De Marco V, Aragona M, Boscolo-Berto R, Cavalleri S, Artibani W, et al. Complications and mortality after radical cystectomy for bladder transitional cell cancer. J Urol. 2009;182(3):914-21.

[13] Hollenbeck BK, Miller DC, Taub D, Dunn RL, Khuri SF, Henderson WG, et al. Identifying risk factors for potentially avoidable complications following radical cystectomy. J Urol 2005; 174:1231-7.

[14] Chang SS, Cookson MS, Baumgartner RG, Wells N, Smith JA, Jr. Analysis of early complications after radical cystectomy: results of a collaborative care pathway. J Urol. 2002;167(5):2012-6. 
[15] Maffezzini M, Campodonico F, Canepa G, Gerbi G, Parodi D. Current perioperative management of radical cystectomy with intestinal, urinary reconstruction for muscle-invasive bladder cancer and reduction of the incidence of postoperative ileus. Surg Oncol. 2008;17(1):41-8.

[16] Cannon WB, Murphy FT. The Movements of the Stomach and Intestines in Some Surgical Conditions. Ann Surg. 1906;43(4):512-36.

[17] Schuster TG, Montie JE. Postoperative ileus after abdominal surgery. Urology 2002;59:465-71.

[18] Jentzmik F, Schostak M, Stephan C, Baumunk D, Lingnau A, Weikert S, et al. Extraperitoneal radical cystectomy with extraperitonealization of the ileal neobladder: a comparison to the transperitoneal technique.World $\mathrm{J}$ Urol. 2010;28(4):457-63.

[19] Serel TA, Sevin G, Perk H, Kooar A, Soyupek S. Antegrade extraperitoneal approach to radical cystectomy and ileal neobladder. Int J Urol 2003;10(1):25-8
[20] Varkarakis IM, Chrisofos M, Antoniou N, Papatsoris A, Deliveliotis C. Evaluation of findings during re-exploration for obstructive ileus after radical cystectomy and ileal-loop urinary diversion: insight into potential technical improvements. BJU Int. 2007;99(4):893-7.

[21] Chang SS, Baumgartner RG, Wells N, Cookson MS, Smith Jr. JA. Causes of increased hospital stay after radical cystectomy in a clinical pathway setting. Journal of Urology 2002;167:208-11.

[22] Kulkarni JN, Agarwal H. Transperitoneal vs. extraperitoneal radical cystectomy for bladder cancer: A retrospective study. Int Braz J Urol. 2018;44(2):296-303.

[23] Wuethrich PY, Vidal A, Burkhard FC. There is a place for radical cystectomy and urinary diversion, including orthotopic bladder substitution, in patients aged 75 and older: Results of a retrospective observational analysis from a high-volume center. Urol Oncol. 2016;34(2):58:19-27. 